\title{
Inner Ear Active Hearing Device in Non-Otosclerotic, Severe, Mixed Hearing Loss
}

\author{
Maurizio Barbara, Luigi Volpini, Edoardo Covelli, Chiara Filippi, and Simonetta Monini \\ Otolaryngology Clinic, NESMOS Department, Medicine and Psychology, Sapienza University, Rome, Italy
}

\begin{abstract}
Objective: To verify the efficacy of a powerful active hearing device in a patient different from far-advanced otosclerosis, specifically when the stapes footplate is mobile. Patient: A patient with severe-to-profound mixed hearing loss, who was not benefiting from the use of a conventional hearing aid, was selected for an inner ear active implant. This was justified by a bone conductive threshold above $60 \mathrm{~dB}$, which had discouraged any other rehabilitative solutions such as a bone conductive implant, or an active middle ear implant (AMEI).
\end{abstract}

Intervention: The hearing device was surgically applied using a combined transmastoid/transcanal approach. During surgery, a mobile stapes were found and was perforated for the insertion of a piston prosthesis, crimped on the newincus of the device.

Main Outcome Measure: The bone conduction threshold was assessed postoperatively to identify any possible surgery-related hearing deterioration. Pure tone audiometry was conducted in a sound field, and a speech reception threshold test was performed with the contralateral ear masked. The hearing outcome was assessed soon after the implant activation (6 weeks after surgery), and 6 months after surgery.

Results: Upon activation of the device, a $\mathrm{PTA}^{4}$ of $45 \mathrm{~dB}$ was obtained (at $0.5,1,2$ and $4 \mathrm{kHz}$ ). At 6 months after surgery, the speech discrimination score reached $90 \%$ at $80 \mathrm{~dB}$ SPL.

Conclusion: The application of the Codacs $^{\mathrm{TM}}$ device has shown to be compatible with a mobile stapes footplate, as demonstrated in this report. The footplate perforation did not cause any further hearing deterioration, and has allowed to achieve a favorable auditory outcome. Key Words: Codacs-Implantable hearing device-Mixed hearing loss.

Otol Neurotol 37:xxx-xxx, 2016.
The auditory rehabilitation of a mixed hearing loss has recently become the target of several implanting centers worldwide. Today, choosing the most appropriate device is mostly on the basis of the bone-conductive (BC) threshold which, up to a certain level $(30-35 \mathrm{~dB})$, could benefit from a $\mathrm{BC}$ device: either conventional, fitted in hearing spectacles, or bone-anchored. This latter system is featured by differently powered processors, the most powerful of which would theoretically be appropriate for $\mathrm{BC}$ thresholds beyond 40 to $45 \mathrm{~dB}$. However, at these levels, recent reports have emphasized the favorable role played by an active middle ear implant (AMEI), either placed on the remnants of the ossicular chain or in contact with the round window membrane (1). Nevertheless, also an AMEI seems to work only up to certain BC levels, for example, $65 \mathrm{~dB}$ at $4 \mathrm{kHz}$ for the Vibrant Soundbridge, showing much less or no efficacy for $\mathrm{BC}$ thresholds beyond these limits. These are, therefore, regarded to be an option for cochlear implants.

Address correspondence and reprint requests to Maurizio Barbara, M.D., Ph.D., Otorhinolaryngology, University Hospital Sant'Andrea,

Via Di Grottarossa 1035, 00189 Rome, Italy;

E-mail: maurizio.barbara@uniroma1.it

The authors report no conflicts of interest.
A new device has recently been introduced for bridging this indication gap, and has initially been applied to the most frequent pathology that causes an auditory condition of this kind, i.e., otosclerosis (2-5). This device has been experimentally shown to possess an extremely powerful actuator, providing a direct acoustic cochlear stimulation that bypasses the anatomical, middle ear conductive apparatus, and delivers the stimulation energy directly to the inner ear fluids (3). Previous literature reports have reported that this device may provide a substantial benefit when the $\mathrm{BC}$ threshold is still measurable, but lies beyond the indication range for a $\mathrm{BC}$ implant or an AMEI.

More recently, this hearing device has been proposed in pathologies or surgical sequels other than otosclerosis. In particular, its application has been associated as a functional, sequential step to a subtotal petrosectomy, in which case one might assume that, even in absence of ossicular remnants, the stapes footplate would mostly be unaffected and therefore mobile (6).

The purpose of the present article is to report a case of application of an inner ear hearing active device on a subject with severe-to-profound mixed hearing loss that did not originate from otosclerosis or from sequels of chronic otitis media, and in whom the stapes was intraoperatively found to be mobile. 


\section{CASE REPORT}

A 46-year-old man presented with bilateral mixed hearing loss of a moderate degree in the right ear, and of a severe degree in the left ear (Fig. 1). He had undergone two previous transcanal ear surgeries on the left ear, performed elsewhere by a single surgeon. No intraoperative findings of stapes fixation were reported, so that the surgeon decided not to proceed with any specific stapedioplasty procedure. However, after the second operation, an audiogram revealed a marked deterioration of the $\mathrm{BC}$ threshold in the operated ear, with a low discrimination score that had initially prompted a rehabilitative attempt with an air-conduction conventional hearing aid. Unfortunately, owing to its poor beneficial effect, the patient had to abandon the hearing aid and looked for alternative rehabilitative solutions. At our implanting center, a Codacs (Cochlear, Melbourne, Australia) device was proposed and its surgical application was performed via a combined transmastoid/transcanal approach (5). As anticipated from the previous surgical reports, the stapes was found to be mobile. So, after its disarticulation from the incus, this latter was removed after using it as a reference for the distance of the Codacs neo-incus actuator to the footplate. Then, the stapes crura were resected with a laser (Revolix jr, Lisa Laser, Katlenburg-Lindau, Germany) at a power of $7 \mathrm{~W}$. Afterwards, the same laser was used to carbonize the footplate at its mid-portion $(2.5 \mathrm{~W})$, regularizing the hole with a manual perforator. A $6.00 \mathrm{~mm}$ long, 0.4-mm wide stapes prosthesis (AudioTechnologies, Gossolengo, Italy) was then anchored to the Codacs new-incus actuator, and then crimped. The immediate postoperative $\mathrm{BC}$ threshold was observed to be unchanged with respect to the preoperative level
(Fig. 1). Six weeks after surgery, at the activation of the device, a PTA ${ }^{4}$ of $45 \mathrm{~dB}$ was obtained in sound field $(250,500,1000$ and $2000 \mathrm{~Hz})$, with the contralateral ear occluded, showing a remarkable improvement over the unaided situation (Fig. 2). The speech discrimination score, obtained whereas the contralateral ear was masked (100 dB SPL), and performed at activation of the device (i.e., 6 weeks after surgery), showed $20 \%$ of discrimination at $80 \mathrm{~dB}$, which improved up to $90 \%$ at the 6-month follow-up control (Fig. 3).

\section{DISCUSSION}

Nowadays, the auditory rehabilitation of severe-toprofound hearing loss is more likely to be obtained via cochlear implantation $(\mathrm{CI})$, including cases that are still presenting with a conductive component, with a poor $\mathrm{BC}$ threshold, and a low discrimination score. The efficacy of a CI in end-stage otosclerosis has also been reported (7). However, in the CI-implanted otosclerotic patients, a higher percentage of facial nerve stimulation can occur, obliging to deactivate some of the electrodes, with an eventual decrease of the auditory improvement. Additionally, from a subjective point of view, one may assume that the perception of an electrical stimulation could be different from the one that is received acoustically. In this respect, recent reports have suggested that in patients of advanced otosclerosis, applying a protocol that consists of a standard stapedotomy and a successive hearing aid could be still advantageous for the patient before proposing a CI $(8,9)$.

For the same reason, a powerful inner ear active implant has been auspicated for these advanced otosclerotic patients, and has so far been applied in a discreet number of patients at selected otologic implanting centers (2-5).
PRE-OP AUDIOGRAM

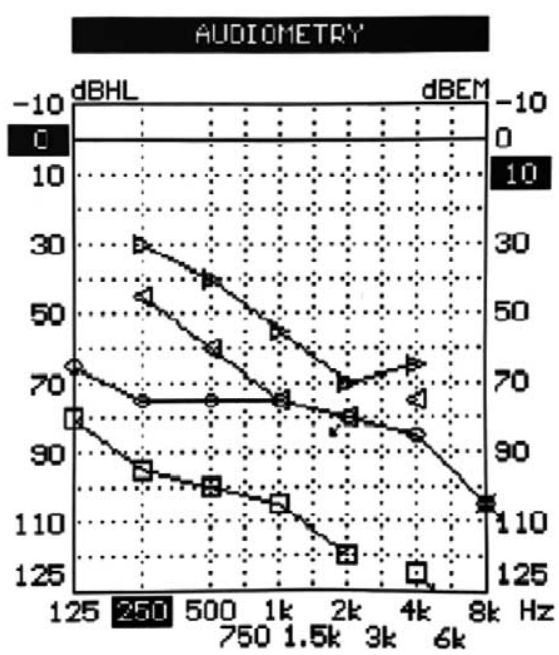

POST-OP BONE CONDUCTION THRESHOLD

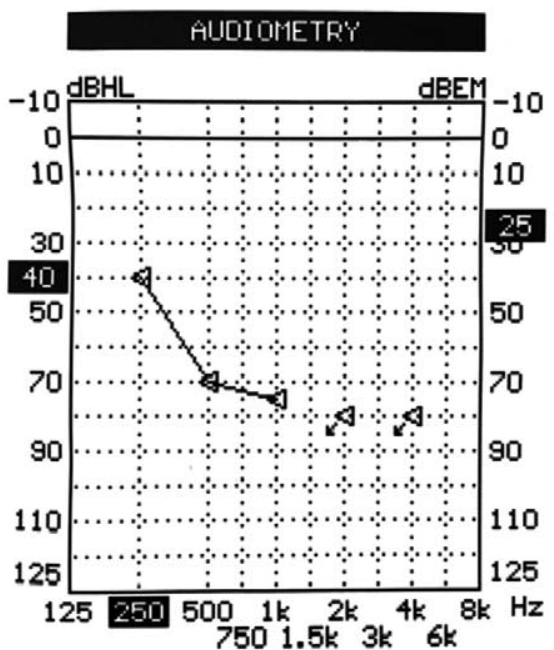

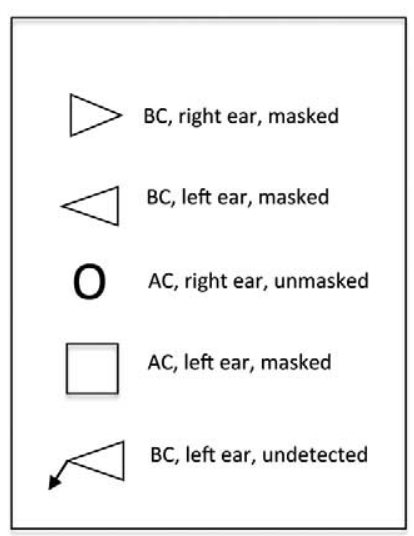

FIG. 1. Left, Preoperative pure tone audiogram of the Codacs candidate; right, immediate postoperative bone conduction threshold of the Codacs implanted patient. AC indicates air conduction; BC, bone conduction. 

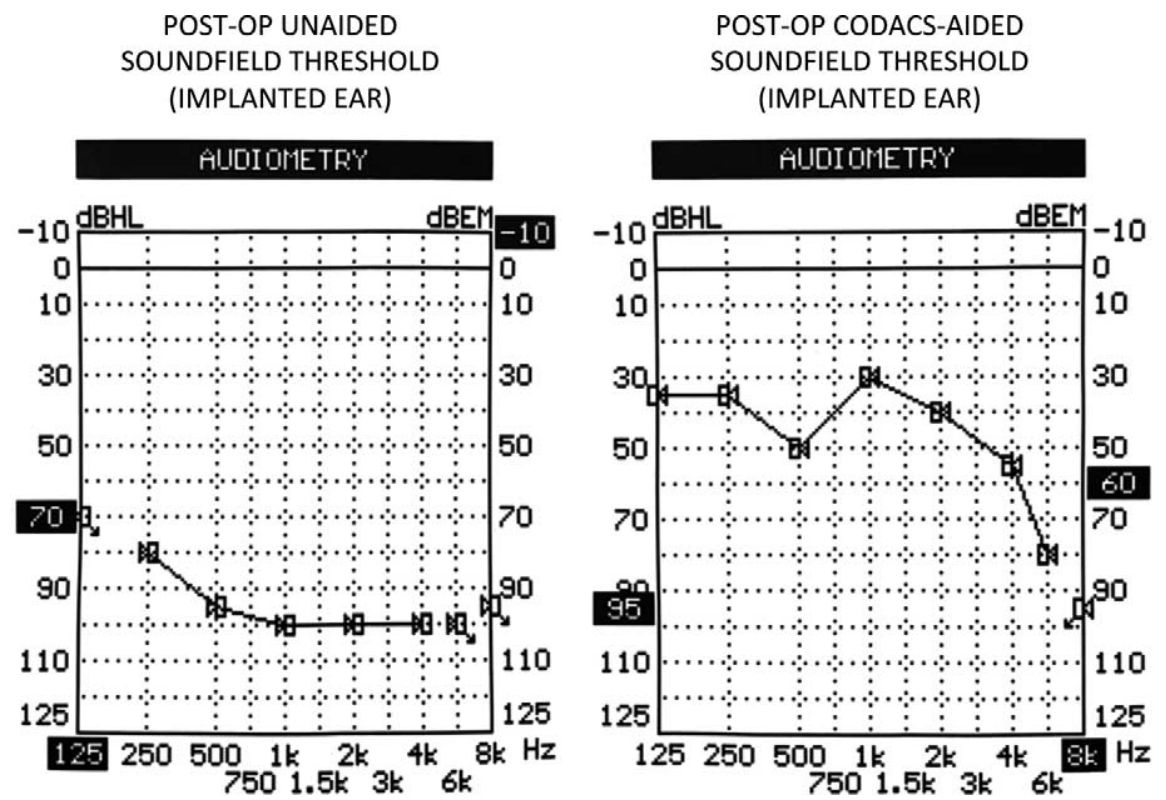

FIG. 2. Left, unaided sound field pure tone threshold of the Codacs implanted patient; right, Codacs-aided sound field pure tone threshold of the implanted patient.

From these preliminary clinical experiences, it has been demonstrated that it provides a high-powered electromechanical stimulation, and as clear and natural a sound as one would expect from an acoustic stimulation. However, it cannot yet be predicted whether the progression of otosclerotic disease will later on necessitate a CI.
From a surgical point of view, in these otosclerotic patients, the handling of the stapes footplate strictly resembles what is performed in conventional stapes surgery. As such, its perforation can be performed via manual instruments, electric drills, or lasers. The footplate manipulation in non-otosclerotic patients is,
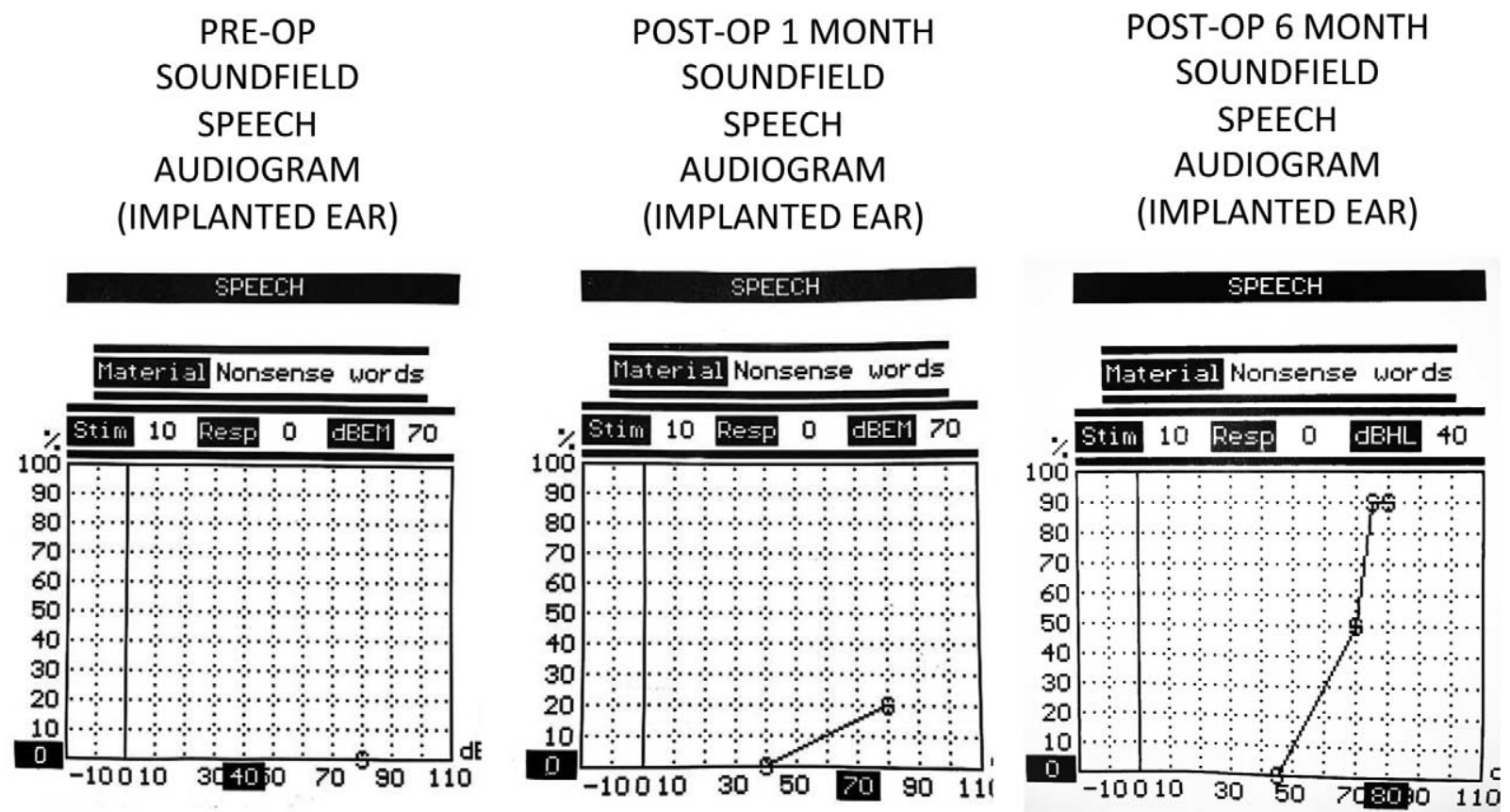

FIG. 3. Left, unaided postoperative speech audiogram of the Codacs implanted patient; middle, the speech audiogram at activation of the device, 6 weeks after surgery, showed a $20 \%$ of discrimination at $80 \mathrm{~dB}$ of stimulation, with the contralateral ear masked; right, the speech audiogram performed 6 months after surgery, showed a $90 \%$ of discrimination at $80 \mathrm{~dB}$ of stimulation, with the contralateral ear masked. 
contrarily, considered a potential hearing-threatening maneuver.

A few years ago, Fisch et al. (10) proposed treating these patients with conductive hearing loss and an absent incus, with the positioning of a piston prosthesis crimped on an artificial titanium rod (neo-malleus) inserted through the reconstructed eardrum. This procedure could also include perforation of a nearly normal and mobile stapes. Although it has not been popularized, the author demonstrated that footplate perforation and placement of a stapes prosthesis in patients with a mobile footplate were feasible, without running greater risks to the inner ear in otosclerotic patients. Some similarities can easily be found between the passive Fisch prosthesis and the inner ear active implant presented in this report. The latter is in fact implemented with an artificial rod tip (new-incus), on which a piston prosthesis is crimped, that acts as the actuator for an acoustic, electromechanical stimulation.

The patient of the present report was offered the opportunity to receive an active inner ear device owing to his hearing impairment that was not having any benefit from a conventional hearing aid, and to his initial refuse to undergo CI surgery. The progression of his hearing loss still remains controversial. In fact, initially, history was in favor of the presence of an ossicular fixation although stapes fixation was not found during the two previous surgeries. Whether the cochlear deterioration, expressed by the low bone conduction threshold, was due to the original pathology or to the previous surgeries is also not very clear. As a matter of fact, during the present surgical procedure, a mobile stapes was encountered, needing two separate laserassisted maneuvers: first, the removal of the crura, then the perforation of the footplate. Despite the possible risks related to the manipulation of a mobile stapes, the early postoperative assessment revealed an unchanged $\mathrm{BC}$ threshold, in agreement with what has earlier been described by Fisch et al. (10) with a passive prosthesis, and with the recent report of sequential application after subtotal petrosectomy (6). It further needs to be elucidated whether this device could also be indicated to the failures from stapes surgery that end up with a deterioration of the $\mathrm{BC}$ threshold beyond the indication range of a $\mathrm{BCI}$ or an AMEI.

It is possible to conclude that the present inner ear active hearing device represents a powerful tool that enables a significant hearing improvement not only in patients of otosclerosis but also when the stapes footplate is mobile. This device could, therefore, be used for patients with severe-to-profound, mixed hearing loss, as an intermediate option between stapedotomy/conventional hearing aid and CI.

\section{REFERENCES}

1. Mojallal H, Schwab B, Hinze AL, Giere T, Lenarz T. Retrospective audiological analysis of bone conduction versus round window vibratory stimulation in patients with mixed hearing loss. Int $J$ Audiol 2015;54:391-400.

2. Lenarz T, Zwartenkot JW, Stieger C, et al. Multicenter study with a direct acoustic cochlear implant. Otol Neurotol 2013;34:1215-25.

3. Zwartenkot JW, Snik AF, Mylanus EA, Mulder JJ. Amplification options for patients with mixed hearing loss. Otol Neurotol 2014; $35: 221-6$

4. Lenarz T, Verhaert N, Desloovere C, et al. A comparative study on speech in noise understanding with a direct acoustic cochlear implant in subjects with severe to profound mixed hearing loss. Audiol Neurootol 2014;19:164-74.

5. Lenarz T, Schwab B, Maier H, Kludt E. Direct acoustic cochlear stimulation for therapy of severe to profound mixed hearing loss: Codacs $^{\mathrm{TM}}$ Direct Acoustic Cochlear Implant System. HNO 2014;62:481-9.

6. Schwab B, Kludt E, Maier H, Lenarz T, Teschner M. Subtotal petrosectomy and Codacs: new possibilities in ears with chronic infection. Eur Arch Otorhinolaryngol 2015. [Epub ahead of print].

7. Semaan MT, Gehani NC, Tummala N, et al. Cochlear implantation outcomes in patients with far advanced otosclerosis. Am J Otolaryngol 2012;33:608-14.

8. van Loon MC, Merkus P, Smit CF, Smits C, Witte BI, Hensen EF. Stapedotomy in cochlear implant candidates with far advanced otosclerosis: a systematic review of the literature and meta-analysis. Otol Neurotol 2014:35:1707-14.

9. Kabbara B, Gauche C, Calmels MN, et al. Decisive criteria between stapedotomy and cochlear implantation in patients with far advanced otosclerosis. Otol Neurotol 2015;36:e73-8.

10. Fisch U, May JS, Linder T. Tympanoplasty, Mastoidectomy, and Stapes Surgery. Stuttgart, Germany: Thieme; 2011. 